\title{
A Guide to Visualizing Trajectories of Change with Confidence Bands and Raw Data
}

\author{
Andrea L. Howard \\ Department of Psychology, Carleton University
}

This manuscript was accepted for publication at Advances in Methods and Practices in Psychological Science on August 26, 2021. This preprint is the peer-reviewed accepted version but has not yet been copyedited and may differ from the final version published in the journal.

\author{
Author Note \\ Andrea L. Howard https://orcid.org/0000-0002-9843-9577 \\ Correspondence concerning this article should be addressed to Andrea L. Howard, \\ Carleton University, 1125 Colonel By Drive, Ottawa, ON K1S 5B6. Email:
} andrea.howard@carleton.ca 


\begin{abstract}
This tutorial is aimed at researchers working with repeated measures or longitudinal data who are interested in enhancing their visualizations of model-implied mean-level trajectories plotted over time with confidence bands and raw data. The intended audience is researchers who are already modeling their experimental, observational, or other repeated measures data over time using random effects regression or latent curve modeling, but who lack a comprehensive guide to visualize trajectories over time. This tutorial uses an example plotting trajectories from two groups, as seen in random effects models that include time $\times$ group interactions and latent curve models that regress the latent time slope factor onto a grouping variable. This tutorial is also geared toward researchers who are satisfied with their current software environment for modeling repeated measures data but who want to make graphics using R software. Prior knowledge of $\mathrm{R}$ is not assumed, and readers can follow along using data and other supporting materials available via OSF at https://osf.io/78bk5/. Readers should come away from this tutorial with the tools needed to begin visualizing mean trajectories over time from their own models and enhancing those plots with graphical estimates of uncertainty and raw data that adhere to transparent practices in research reporting.
\end{abstract}




\section{A Guide to Visualizing Trajectories of Change with Confidence Bands and Raw Data}

Repeated measures research designs and data analyses that leverage longitudinal data are increasingly prevalent in psychology. In Psychological Science, 19.2\% of research articles published between 2015 and 2020 included the keyword "longitudinal," compared to just 7.0\% of research articles published between 1995 and 2000. Advances in the latter part of the $20^{\text {th }}$ century introduced alternatives to repeated measures ANOVA and the popularization of techniques to fit curves or trajectories to longitudinal data using structural equation models and random effects (multilevel) regression (e.g., Bryk \& Raudenbush, 1987; Meredith \& Tisak, 1990). Instead of simple mean differences at varying times of measurement, models with trajectories summarize underlying patterns of change over time, typically using polynomial (e.g., linear, quadratic) or spline functions fitted to repeated measures data. Fitted trajectories are popular in life course studies tracking age-related changes over years, but also frequently appear in experimental research (e.g., impact of a self-reflection manipulation on patterns of change in emotionality; Dorfman et al., 2021), short-term observational studies (e.g., daily diaries tracking change in emotion regulation in the days leading up to and following a major sports competition; Tamminen et al., 2019), and physiological measures (e.g., effects of stress on diurnal cortisol over hours since waking; Young et al., 2019).

Research questions arising in repeated measures designs often focus on evaluating patterns of mean-level change in a behavior over time. On average, does a behavior systematically increase, decrease, or change direction? A common practice to aid in interpreting patterns of change is to derive predicted values or point estimates from the model fixed effects and visualize the trajectories by plotting values at various increments of time. There is often an accompanying hypothesis that change over time differs conditional on a grouping variable or time-invariant factor (e.g., experimental condition, personality trait level, gender), and plots are 
often constructed to showcase the trajectories of two or more groups at a time. Most software for longitudinal analysis includes built-in functionality to create visual displays of fitted trajectories from the fixed effects, but these are not typically accompanied by displays of raw data nor estimates of uncertainty around those trajectories. Psychologists' appetite for transparent research practices is rapidly increasing, and a key recommendation in data visualization is to include confidence intervals around point estimates (Cumming, 2014). For simple group mean comparisons, these are easily estimated with popular commercial software such as SPSS. Techniques and tools for computing confidence bands-confidence intervals surrounding a continuous range of point estimates — are not as readily available nor widely documented online. When user-friendly tools are available, they may not apply to more complex models typically used for repeated measures data such as random effects regression (as is currently the case for the predict() function in R). The goal of this tutorial is to demonstrate a general-purpose technique for computing confidence bands around mean-level fitted trajectories of repeated measures data modeled in any software. The approach illustrated in this tutorial is meant to improve the transparency of the research data presented in publications and the conclusions that can be drawn from models of change over time. Although the technique shown here focuses on longitudinal data, it can be applied more broadly, including to multilevel designs with crosssectional data where there might be an interest in visualizing uncertainty around predicted values. All demonstrations are presented in R software (R Core Team, 2019), and are suitable for visual displays of original analyses performed in any software environment.

\section{Data Example Used in This Tutorial}

Longitudinal or repeated measures data take many forms, appearing in experimental, observational, time series, and psychophysiology study designs. A common theme anchoring these diverse designs is researchers' interest in identifying and visualizing trends modeled over 
time in their data. What differs over studies is the functional form of time in the model: The simplest models include one fixed effect, slope, or vector of factor loadings representing a single linear rate of change, but models commonly include two or more fixed effects capturing polynomial functions of change or linear splines.

The example I chose for this tutorial strikes a balance across the diverse designs of interest in psychological science. Drawing on a model study by Tamminen and colleagues (2019), example data presented here comprise a simulated sample of $n=60$ competitive athletes surveyed once per day over 10 days. Surveys completed on the first five days measured interpersonal emotion regulation on practice days leading up to a major competition, which took place on the sixth day. Surveys completed on the sixth through tenth days measured interpersonal emotion regulation after competition. In their original article, Tamminen et al. describe research showing that athletes on teams interact with each other in ways that deliberately impact each others' emotions, cognitions, and motivation for competition. They found that athletes' attempts to worsen their teammates' moods - for example, by ignoring them or trying to embarrass them-declined precipitously in the days leading up to competition and leveled off or increased in the days following competition.

The pattern described above implies a question about two-part trajectories for athletes: what are the rates of change in attempts to worsen teammates' moods in the days before and after a major team competition? The data for this tutorial are available at https://osf.io/78bk5/ and include an ID number for each simulated athlete, a variable indicating whether the athlete's team won or lost their competition ("loss", coded 0 or 1, respectively), an index of time for each athlete ("time", ranging from 1 to 10), and a score indicating athletes' attempts to worsen teammates' moods each day, where higher values reflect greater attempts to worsen mood. I used these data to fit 10-day trajectories for athletes whose teams won versus lost their competitions. 
All demonstrations presented here generalize to repeated measures studies with any number of time points measured at any intervals and on any time scale (e.g., minutes, hours, weeks, years).

\section{Following Along in R Software}

The code and descriptions to follow presume that readers have already installed a copy of R software (freely available here: https://cran.r-project.org; also frequently used with the free RStudio IDE interface: https://www.rstudio.com/products/rstudio/download) updated to a recent version (3.6.2 or higher ${ }^{1}$ ). No prior experience with $\mathrm{R}$ is necessary to follow the demonstrations below, though some prior experience with R may be helpful. Demonstrations presume that readers are familiar with typical tabular results of a growth model comprising an intercept, slopes, and effects of a time-invariant predictor on those slopes.

Data provided with this tutorial can be used in any software package. If you have downloaded the file sportdata. CSV provided with this tutorial and you are reading data into $\mathrm{R}$ for the very first time, use the code below to name a new data object called athlete and assign it (with the <- assignment operator) the contents of a file from your computer. The file.choose( ) option will prompt R to open a file navigator (Finder or Explorer) window for you to select a file:

athlete $<-$ read.csv $($ file $=$ file. $\operatorname{choose}())$

$\mathrm{R}$ software includes a wide range of built-in base functions, but there are also numerous third-party supplemental functions called packages that need to be loaded into the $\mathrm{R}$ environment each time the software is restarted before they can be used. Key packages used in this tutorial are: the dplyr (Wickham et al., 2021) and ggplot2 (Wickham, 2016), packages belonging to the larger tidyverse suite of packages for managing, manipulating, plotting, and programming data in

\footnotetext{
1 The commands in this tutorial were verified functional in R version 4.1.0 and RStudio version 1.4.1717
} 
$\mathrm{R}$ (https://www.tidyverse.org), and viridis (Garnier, 2018) to control plot colors. To install the packages (do this only once), use the following commands:

install.packages("dplyr")

install.packages("ggplot2")

install.packages("viridis")

To load the packages (do this every time you restart R), call each package with a library() command:

library (dplyr)

library(ggplot2)

library(viridis)

Readers working primarily outside of $\mathrm{R}$ are encouraged to first run the model from the data provided in their preferred software environments (e.g., SPSS, SAS, STATA, or Mplus). Readers already working in $\mathrm{R}$ or interested in doing so can fit these data using the $\operatorname{lmer}($ ) function from the lme4 package (Bates et al., 2015) and companion lmerTest package (Kuznetsova et al., 2017) to obtain hypothesis tests. To model the proposed two-part trajectories - attempts to worsen teammates' moods in the days before and after a major team competition - some data management is needed to create codes to represent these separate but additive linear effects. This technique is commonly used in spline regression to fit segmented lines to nonlinear data (Marsh \& Cormier, 2002). In longitudinal analyses, and commonly in latent growth curve modeling, this approach is known as piecewise growth (Flora, 2004).

In $R$, we can use the ifelse( ) function to create coded values representing rate of change in days before competition, and it reads as follows: if "time" in the data athlete is less than or equal to 5, assign a value of "time" minus 5, otherwise assign a value of zero. These recodes are assigned to a new variable before in the data athlete.

athlete\$before <- ifelse(athlete\$time <= 5, athlete\$time-5, 0 )

athlete\$after <- ifelse (athlete\$time >5, athlete\$time-5, 0 ) 
The parenthetical command creating a new variable after to represent change in days after competition reads in a similar fashion, isolating instead "time" greater than 5 . Using the head ( ) command to show the first 10 values, we see data for participant id \#1, the original values of time, their score each day, and new coded values for change before and after competition day:

\begin{tabular}{|c|c|c|c|c|c|c|}
\hline \\
\hline \multicolumn{7}{|c|}{$\begin{array}{l}\text { head(atnlete, } n=10) \\
\text { id time loss score before after }\end{array}$} \\
\hline 1 & 1 & 1 & 0 & 3.81 & -4 & 0 \\
\hline 2 & 1 & 2 & 0 & 4.17 & -3 & 0 \\
\hline 3 & 1 & 3 & 0 & 3.33 & -2 & 0 \\
\hline 4 & 1 & 4 & 0 & 3.10 & -1 & 0 \\
\hline 5 & 1 & 5 & 0 & 2.66 & 0 & 0 \\
\hline 6 & 1 & 6 & 0 & 2.13 & 0 & 1 \\
\hline 7 & 1 & 7 & 0 & 3.55 & 0 & 2 \\
\hline 8 & 1 & 8 & 0 & 3.22 & 0 & 3 \\
\hline 9 & 1 & 9 & 0 & 3.29 & 0 & 4 \\
\hline 10 & 1 & 10 & 0 & 3.06 & 0 & 5 \\
\hline
\end{tabular}

Using these data, we can set up a random effects regression model predicting athlete's score from group membership (winning team, $l o s s=0$; losing team, loss $=1$ ), the two additive linear functions for change (before and after), and their interactions. The model shown here permits a random intercept and random slopes for both functions of change, but other combinations of random effects are defensible, including random intercept-only models. After loading the appropriate package libraries for the analysis, we call the 1 mer ( ) function and define a model in which score is predicted from the linear combination of before, after, loss, and their interactions (before*loss and after*loss). Random effects are inside the parentheses; 1 stands for the random intercept and the clustering variable id is denoted after the vertical pipe. By default, this model performs restricted maximum likelihood estimation (REML). The model result is assigned to a model object $\mathrm{m}$ : 


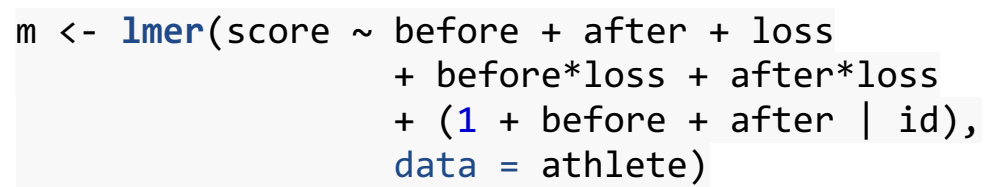

Calling the summary ( ) function for the model object $m$ displays model convergence information, random effects, and fixed effects, the latter of which are shown below:

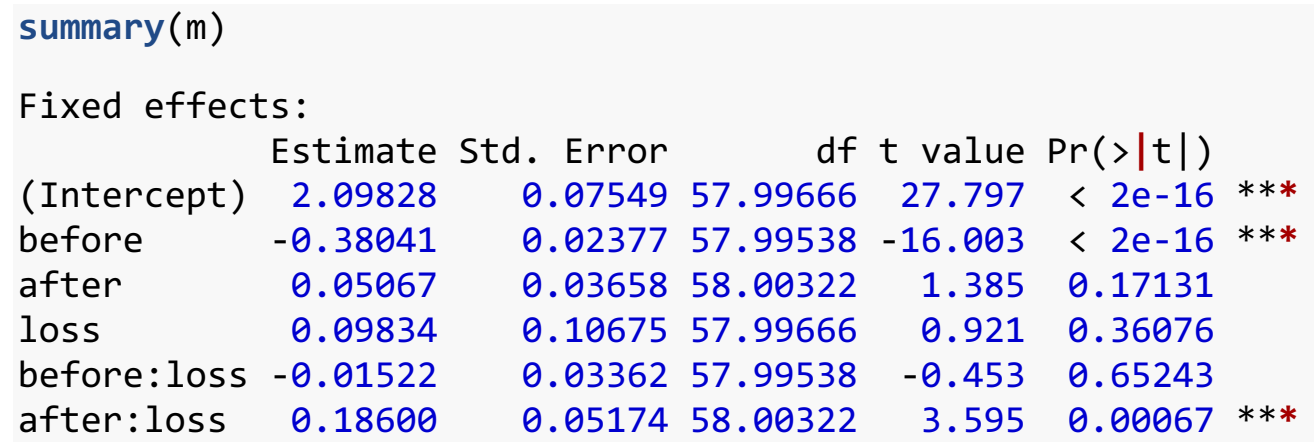

Degrees of freedom for this model were calculated using the default Satterthwaite method (1941), a frequently-used and conservative choice especially in small samples (Luke, 2017; Li \& Redden, 2015). Readers estimating the above model in other software should obtain similar fixed effects and standard errors, but degrees of freedom may differ or may not be given if a $z$-ratio is used instead of a $t$-ratio (as in Mplus software). These differences will affect the choice of critical value for forming confidence bands later in this tutorial. Our practical demonstration begins after reviewing the structure of fitted trajectory plots and how uncertainties around points on a line are computed.

\section{Plotting Fitted Trajectories}

Table 1 shows the fixed effects from the above model predicting athletes' attempts to worsen teammates' moods over 10 days, with separate effects for the time trend in the days before and days after competition. Mean trajectories are also expected to differ for athletes whose teams won versus lost their competitions, and this is captured in the model by interactions between the indicator variable "loss" and the two different time trends. 
VISUALIZING TRAJECTORIES

\section{Table 1}

Fixed effects for the model predicting change over days in athletes' attempts to worsen their teammates' mood before and after a competition day

\begin{tabular}{lcc}
\hline & Estimate & \multicolumn{1}{l}{ SE } \\
\cline { 2 - 3 } Intercept & 2.098 & 0.075 \\
Days before competition & -0.380 & 0.024 \\
Days after competition & 0.051 & 0.037 \\
Loss & 0.098 & 0.107 \\
Days before x Loss & -0.015 & 0.034 \\
Days after x Loss & 0.186 & 0.052 \\
\hline
\end{tabular}

Creating a plot of the average trajectories across individuals from a random effects regression or latent curve model — not including the confidence bands around those averages—is straightforward because all the necessary information is contained in the table of coefficients or parameter estimates of the model's fixed effects. The fixed effects in Table 1 can be rewritten as a linear equation:

$$
\begin{array}{r}
\hat{Y}=2.098-.380(\text { before })+.051(\text { after })+.098(\text { loss }) \\
-.015(\text { before } \times \text { loss })+.186(\text { after } \times \text { loss })
\end{array}
$$

For the trajectories of athletes whose teams won $($ loss $=0)$ and lost $(\operatorname{loss}=1)$, we can find fitted values for a plot by first writing a reduced equation for each trajectory and simplifying then gathering terms:

$$
\begin{aligned}
\hat{Y}_{(\text {loss }=0)}=2.098- & .380(\text { before })+.051(\text { after })+.098(0) \\
& -.015(\text { before } \times 0)+.186(\text { after } \times 0) \\
\hat{Y}_{(\text {loss }=0)}=2.098- & .380(\text { before })+.051(\text { after })
\end{aligned}
$$




$$
\begin{aligned}
\hat{Y}_{(\text {loss }=1)}=2.098- & .380(\text { before })+.051(\text { after })+.098(1) \\
& -.015(\text { before } \times 1)+.186(\text { after } \times 1) \\
\hat{Y}_{(\text {loss }=1)}=2.196- & .395(\text { before })+.237(\text { after })
\end{aligned}
$$

Equations 2 and 4 substitute values of 0 and 1 representing the effect of loss and its role in interaction terms to obtain simple equations for athletes who won (Equation 3) and lost (Equation 5) their competitions. Fitted trajectories can now be computed by substituting into Equations 3 and 5 the values that we used to code days before competition and days after competition in the model. Table 2 shows fitted values for trajectories corresponding to athletes whose teams won and lost their competitions at the midpoint of the study.

\section{Table 2}

Coded values for indices of time representing days before and after competition and average predicted values of attempts to worsen teammates' moods for trajectories corresponding to athletes whose teams won (Equation 3; Fit WIN) or lost their competition (Equation 5; Fit LOSS).

\begin{tabular}{lcccc}
\hline Day & Before & After & Fit WIN & Fit LOSS \\
\cline { 2 - 5 } 1 & -4 & 0 & 3.62 & 3.78 \\
2 & -3 & 0 & 3.24 & 3.38 \\
3 & -2 & 0 & 2.86 & 2.99 \\
4 & -1 & 0 & 2.48 & 2.59 \\
5 & 0 & 0 & 2.10 & 2.20 \\
6 & 0 & 1 & 2.15 & 2.44 \\
7 & 0 & 2 & 2.20 & 2.68 \\
8 & 0 & 3 & 2.25 & 2.92 \\
9 & 0 & 4 & 2.30 & 3.16 \\
10 & 0 & 5 & 2.35 & 3.40 \\
\hline
\end{tabular}


Plotting the scores for each scenario, Figure 1 shows simple fitted trajectories over 10 days, with competition day occurring after the Day 5 assessment and before the Day 6 assessment.

\section{Figure 1}

Simple mean trajectories of attempts to worsen teammates' moods for athletes who won and lost their games on competition day.

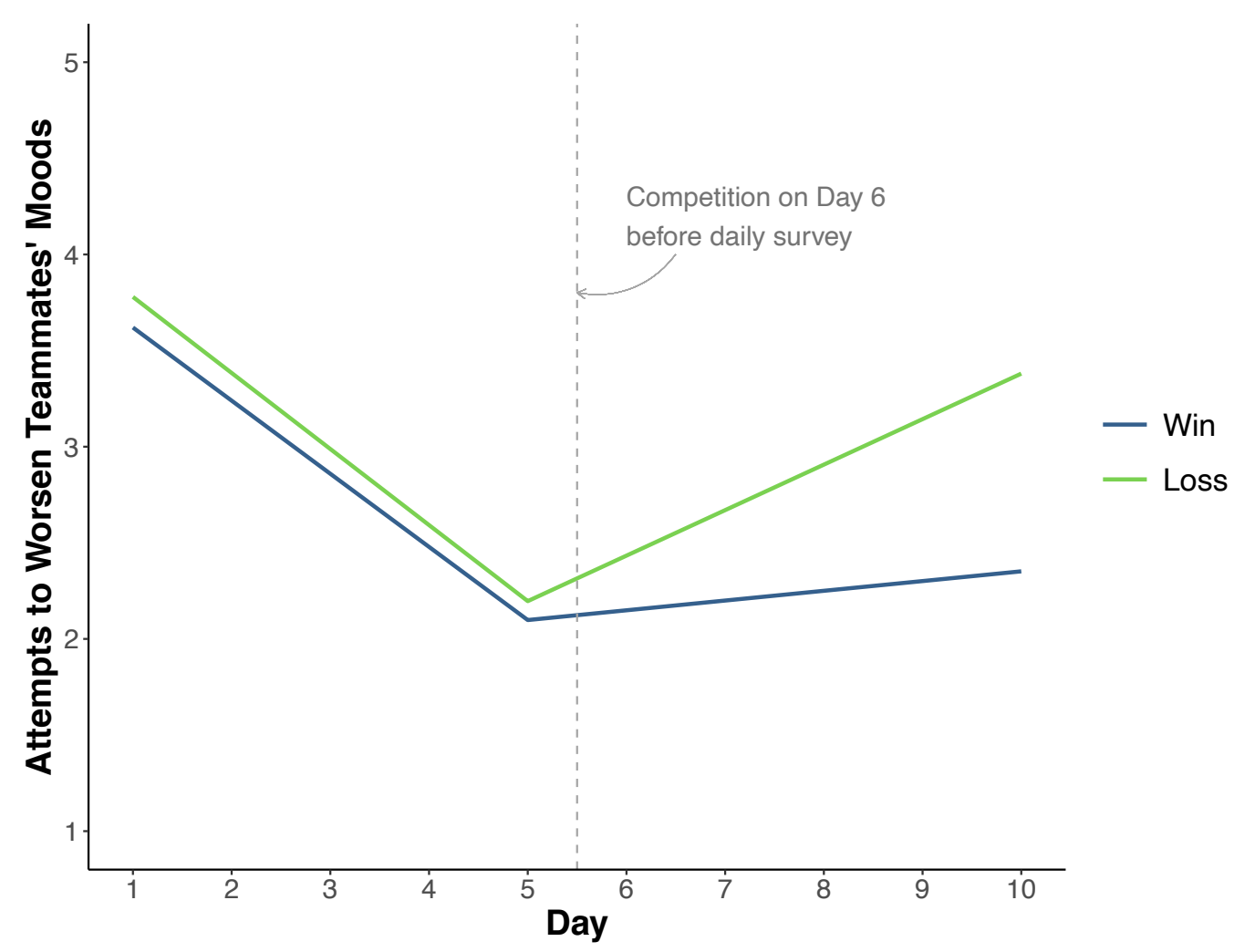

Plots like the one shown in Figure 1 often appear in empirical articles illustrating trajectories of change over time. They are easy to create and require no special software. Fitted values can be computed by hand from the table of fixed effects and plotted in Excel or other spreadsheet software. Notably absent from Figure 1, however, is any index of uncertainty around the lines for each group. The lack of a statistically significant before $\times$ loss interaction indicates that there is insufficient evidence to conclude that the two types of athletes differ in their rates of change in the days leading up to competition. In Figure 1, all athletes appear to reduce their 
attempts to worsen teammates' moods at roughly the same rate. In contrast, the significant after $\times$ loss interaction suggests that attempts to worsen teammates' moods increase for the athletes whose teams lost their competitions and remain fairly low for athletes whose teams won their competitions. We have some confidence that athletes accelerate their attempts to worsen teammates' moods more quickly after a loss than after a win, but at what point are athletes from winning versus losing teams distinguishable? The point estimates appear notably different on Day 10 but still similar on Day 6. Fitted trajectories alone lack the necessary information to draw these kinds of narrower conclusions and we require additional information not included in default software output to visualize uncertainty at individual points along each simple slope.

\section{Uncertainty Around Fitted Trajectories}

Equations 3 and 5 give the simple intercept and simple slopes of the rates of change over time before and after competition day for athletes whose teams won and lost their competitions. Hypothesis tests of these conditional effects are often of interest but require their own composite standard errors. Formulas for calculating such composite standard errors in the regression context were introduced decades ago (Rogosa, 1980; see also Aiken \& West, 1991, pp. 24-26) but popularized more recently with the introduction of web-based software for regression ${ }^{2}$, multilevel regression, and latent curve analysis (Bauer \& Curran, 2005; Preacher, Curran, \& Bauer, 2006). Formulas for obtaining standard errors of simple slopes are special cases of a more general matrix algebra expression for obtaining the variance of any predicted value of interest in the model, also known as the delta method (e.g., Ogasawara, 1999, pp. 107-110; Raykov, 2002). This general procedure requires estimates of variances and covariances among the fixed effects in a given model, contained in an asymptotic covariance matrix (ACOV).

\footnotetext{
${ }^{2}$ For simple linear regression, the interactions package in $\mathrm{R}$ (Long, 2019) is an excellent recently-developed tool for making graphics that include raw data and uncertainty around fitted slopes, among other features.
} 
The ACOV for the model shown in Table 1 is available in the file acov. Csv with the supplemental materials for this tutorial (https://osf.io/78bk5/). For readers who performed the analysis in R using the lmer ( ) function, the ACOV matrix is obtained from the model object $m$ using the vcov( ) command and assigned to a new object acov, as follows:

$\operatorname{acov}<-\operatorname{vcov}(m)$

For readers performing longitudinal analyses in other software (e.g., SPSS, STATA, Mplus), see the supplemental files (https://osf.io/78bk5/) for instructions on obtaining an ACOV matrix and reading it into $\mathrm{R}$. For the purposes of facilitating the present demonstration, the ACOV matrix for this model is also given in the supplemental files and can be read directly into $\mathrm{R}$ as a matrix using the following command:

acov <- as.matrix (read.csv $($ file $=$ file.choose ()$))$

Diagonal elements of the ACOV matrix are the variances of the six fixed effects in Table 1. Taking their square roots gives the standard errors shown in Table 1. Off-diagonal elements of the ACOV matrix are the pairwise covariances between fixed effects. Both variances and covariances are needed to approximate the uncertainty around a given point estimate in a model. An exception is the model intercept, which itself is a point estimate of the outcome variable when all values of $\mathrm{X}$ in the model equal zero. The overall model intercept in Table 1 is 2.098 with a standard error of 0.075 . This information alone is enough to calculate a confidence interval around the estimate of attempts to worsen teammates' moods for athletes from winning teams $($ loss $=0$ ) on day 5 (where before $=0$ and after $=0$ ). A common regression "hack" for obtaining standard errors of point estimates is to recenter $\mathrm{X}$ variables around different, desired values to coerce the intercept to take on a different, specified predicted value with its standard error now reflecting the uncertainty at that new value. With more than a handful of desired point 
estimates and standard errors, however, it becomes much more efficient to use the ACOV matrix as shown in the demonstration that follows.

Standard errors of fitted values. Returning to Table 2, suppose we are interested in obtaining a standard error on Day 7 for an athlete whose team won their competition. We can first define a row vector of $\mathrm{X}$ values that represent this point in the data:

\begin{tabular}{rrrrrr}
\hline Intercept & before & after & loss & before $x$ loss & after x loss \\
1 & 0 & 2 & 0 & 0 & 0 \\
\hline
\end{tabular}

For this test case, values of all variables in the model are represented, including the intercept which always takes a value of 1 . In $R$, the row vector can be created by hand with the cbind( ) function (stands for "column bind"), as follows:

vec $<-\operatorname{cbind}(1,0,2,0,0,0)$

The six values in this vector will be multiplied using matrix operations ${ }^{3}$ with the entire 6 x 6 ACOV matrix. To combine this vector with the ACOV matrix we obtained earlier, we premultiply then postmultiply acov by vec and its transpose:

vec $\% * \%$ acov $\% * \%$ t (vec)

The result of this operation is a single value, 0.013 , the variance of the point estimate of attempts to worsen teammates' mood on Day 7 for an athlete whose team won their competition. The standard error is then the square root of this value, 0.114. A simple confidence interval around the point estimate (Table 2, Day 7, Fit WIN $=2.20)$ is then $2.20 \pm .114 \times t_{\text {crit }\left(\frac{\alpha}{2}\right)}$, where the critical value of $t$ for the present case given $\alpha=.05$ and Satterthwaite $d f=58$, is 2.00 . The lower and upper 95\% confidence limits of the point estimate are 1.97 and 2.43 . Figure 2 repeats Figure 1, with the point estimate and confidence interval for Day 7 highlighted.

\footnotetext{
3 The OSF page for this project (https://osf.io/78bk5) includes a 2-page guide to some matrix algebra basics intended for readers that are less or unfamiliar with matrix operations.
} 


\section{Figure 2}

Simple Mean Trajectories of Attempts to Worsen Teammates' Moods for Athletes who Won and Lost their Games on Competition Day, with Day 7 Confidence Interval Highlighted.

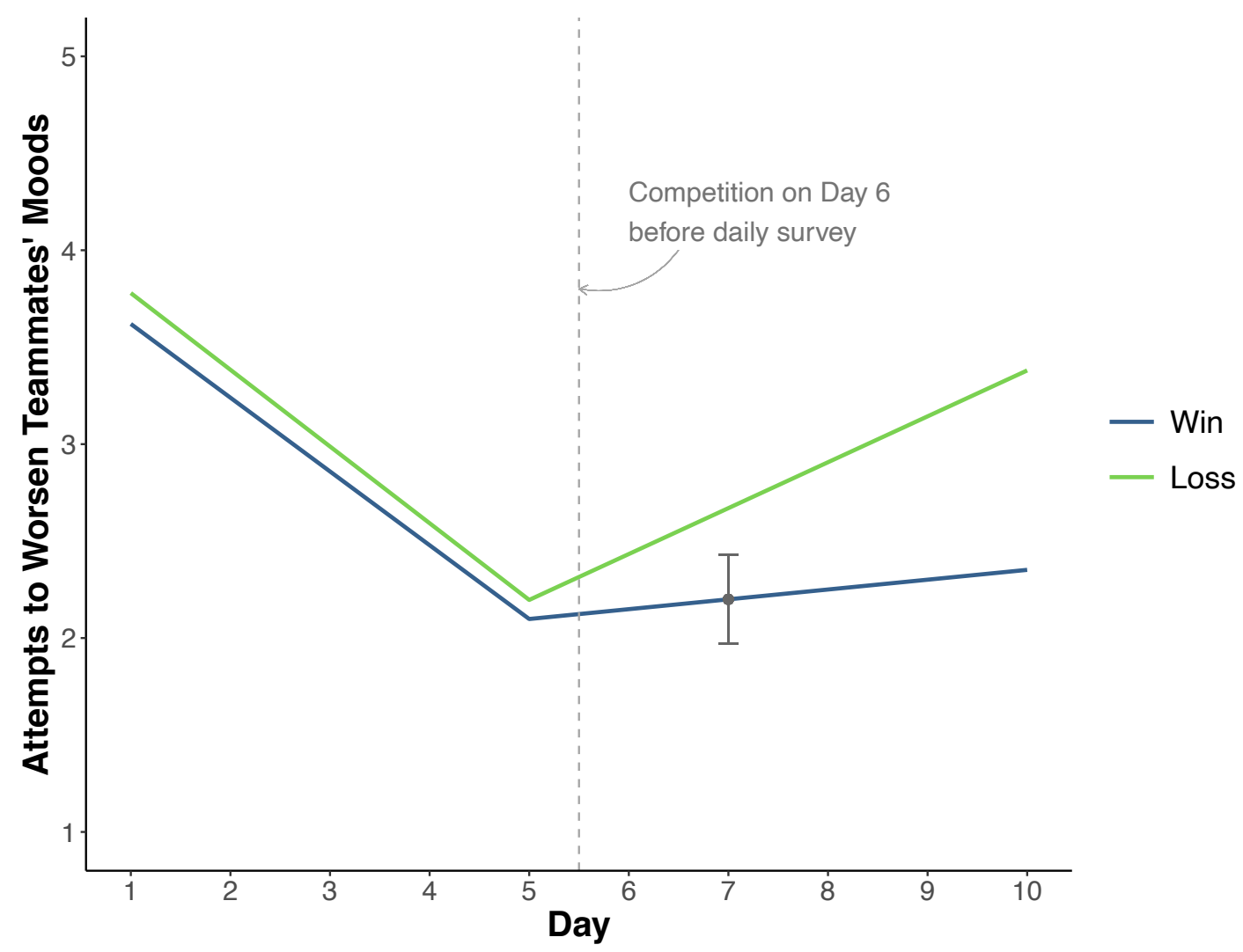

Adding the confidence interval for this single point estimate clarifies that on Day 7, athletes whose teams won their competition engage in attempts to worsen teammates' moods that are less than the average for athletes on Day 7 whose teams lost their competition. This technique is easily extended to calculate standard errors and confidence intervals on all days. Instead of defining a vector of $\mathrm{X}$ values for a single point estimate, we define a matrix of $\mathrm{X}$ values for a range of point estimates. In the present example, we'd like to capture uncertainty at point estimates for athletes whose teams won and lost their competitions, at each of the 10 days in the study. To begin, we can build a data frame containing a series of values for time over which the plotted trajectories are desired and two values of a moderator at which to plot different 
trajectories. In the present example, time is a sequence of 10 equally spaced days, and the moderator is the two coded values for athletes whose teams won (0) and lost (1) their competitions. The code below uses the expand.grid() function to create a dataset pred that contains 20 rows (10 values of time per level of "loss"):

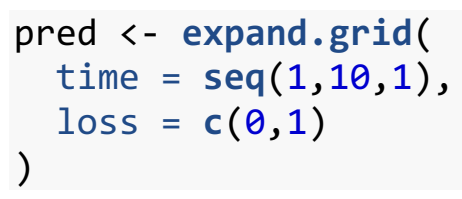

This basic structure can be customized to capture any range of discrete or continuous timepoints and values of time that are evenly or unevenly spaced. In the case of trajectories that differ at values of a continuous moderator we would replace the coded values we used here $(0,1)$ with selected values representing cases that are relatively low and high on the moderator. We might also add more levels of a moderator if three or more trajectories are desired.

As before in the complete data, coded values representing the passage of time before and after competition day can be coded using ifelse( ) function:

pred\$before <- ifelse (pred\$time $<=5$, pred\$time-5, 0 ) pred\$after <- ifelse (pred\$time >5, pred\$time-5, 0 )

Finally, the interactions between loss and the two trajectory components must also be represented in the data, this time using the mutate ( ) function from the previously-loaded dplyr package, including the pipe (\%>) operator, to create two new variables:

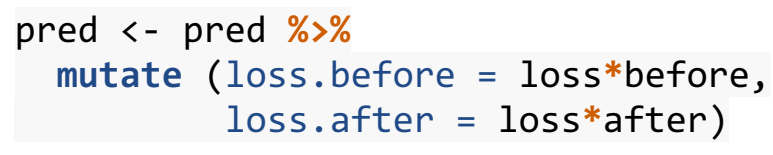

We can view the first few rows of the resulting data as follows:

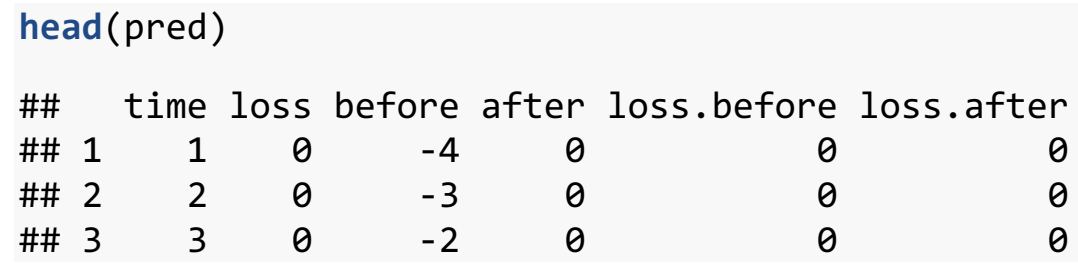




\begin{tabular}{|c|c|c|c|c|c|}
\hline \#\# 4 & 4 & 0 & -1 & 0 & 0 \\
\hline \#\# 5 & 5 & 0 & 0 & 0 & 0 \\
\hline \#\# 6 & 6 & 0 & 0 & 1 & $\theta$ \\
\hline
\end{tabular}

All key predictor variables from the analysis are now represented in this data frame. In a short sequence of steps making use of more functions from the dplyr package, we create a vector of "1"s to stand for the intercept (with the mutate( ) function), put the columns in the order that they appear in both the analysis and in the ACOV matrix (the select()function), convert the data frame to a matrix (the as.matrix() base $\mathrm{R}$ function), and assign the result to a new object xmat:

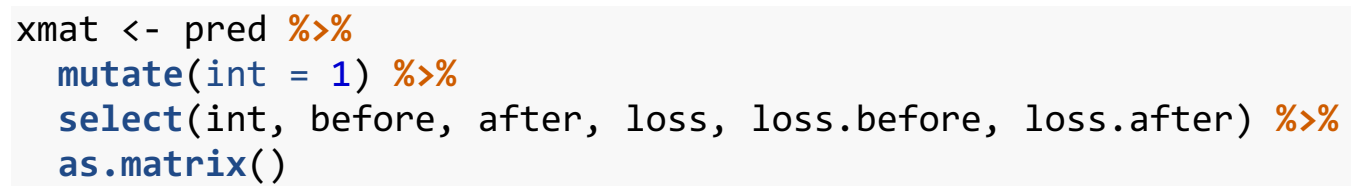

We use xmat to accomplish two tasks: (1) compute predicted values from the model for each row in the data, and (2) calculate standard errors for each of those predicted values.

Predicted values are obtained by multiplying the elements of the data matrix xmat by a vector of model fixed effects like the ones shown in Table 1. For models created outside of the $\mathrm{R}$ environment, these can be read in as data or be entered by hand into a column vector, in the order that they appear in xmat and in acov:

fixed <- rbind $(2.098,-0.380,0.051,0.098,-0.015,0.186)$

For readers performing longitudinal analyses in $\mathrm{R}$ using the $1 \mathrm{me} 4$ package (Bates et al., 2015), the coefficients can be accessed directly by assigning the elements of the column "Estimate" from the coefficients of model object $m$ as follows: fixed <- summary $(m) \$ c o e f f i c i e n t s[$, "Estimate"]

Because the six columns of xmat, (a $20 \times 6$ matrix) appear in the same order as the six elements of fixed (a $6 \times 1$ column vector), multiplying them together will return a column vector of 20 predicted values that we can append to the original data pred as a variable named 
fit for later use in building a plot (and notice the resulting fitted values are identical to those appearing in Table 2):

pred\$fit <- xmat $\% * \%$ fixed

Standard errors are obtained by premultiplying the ACOV matrix by the data matrix xmat, then postmultiplying by the transpose of xmat. This takes a very similar form to the operation we used to find the standard error for a single predicted value. In this case the sequence of operations creates a $20 \times 20$ matrix, of which we retain only the diagonal elements representing the variances of each point estimate (using the diag( ) base R function). We take the square roots of those elements (the sqrt () base R function) and assign the result to a new variable SE in the original data pred for later use in constructing confidence bands.

pred\$SE <- xmat \%*\% acov $\% * \%$ t (xmat) $\%>\%$ $\operatorname{diag}() \%>\%$ sqrt()

Like the example for a single fitted value, we can now compute confidence intervals around each point estimate by multiplying each standard error by a critical value of $t$ (or $z$, or another critical value as demanded by a given model). Using the critical value of 2.00 as before, the lower and upper limits of all 20 point estimates are obtained as follows:

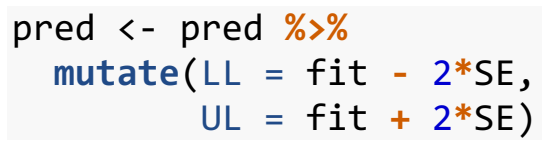

The data are now ready to plot.

\section{Plotting Mean Trajectories with Confidence Bands}

Organizing a plot to communicate as much information as possible in a transparent way without compromising interpretability is in some ways a matter of personal preference and aesthetic skill, but some key elements are important to keep in mind (see Healy, 2019; Tufte, 1983 for guiding principles). These include scaling the axes to avoid distorting the data or 
inflating the apparent magnitude of the results, limiting noninformative elements on a plot (“chartjunk", e.g., background color and prominent gridlines), and using color in a perceptually uniform way that is also visible to people with common forms of colorblindness. The task of transparent and reproducible data visualization is especially easy for plots of fitted trajectories that feature a naturally interpretable, left-to-right ordering of the passage of time on the $\mathrm{x}$-axis (Tufte, 1983). We will apply these principles to create a plot that combines mean trajectories, uncertainty, and raw data while ensuring that all three elements are visible and easy to interpret.

The ggplot2 package for $\mathrm{R}$ is a flexible and customizable platform for making highquality graphics. Plots are built in layers, meaning that a layer containing a fitted trajectory can be overlaid on a layer containing its confidence band. Plots also permit input data from multiple sources, so one layer might visualize the complete raw data while another layer visualizes the smaller set of model-implied mean values of each trajectory.

We can first make a plot containing just the mean trajectories for each athlete group overlaid on their confidence bands. After loading the ggplot2 package, we assign the basic plot specifications using the ggplot ( ) command to a plot object named panelA (identify the data; state which variables appear on the X and Y axes; identify the grouping variable "loss" and call for it to be treated as a discrete Factor). Next we add a line calling the geom_ribbon( ) function to identify the variables containing the lower and upper confidence limits of each fitted value as well as the name of the grouping variable. The confidence band should generally be made semitransparent so any overlaps are visible (here, alpha $=.6$ where $0=$ totally transparent and $1=$ totally opaque). Finally we call the geom_line() function to define the groups for which we have values of the two trajectories and indicate that we would like them to differ in color. For 
now, we're setting aside commands that control the appearance of the plot, such as color choices, background, and axis and legend labels.

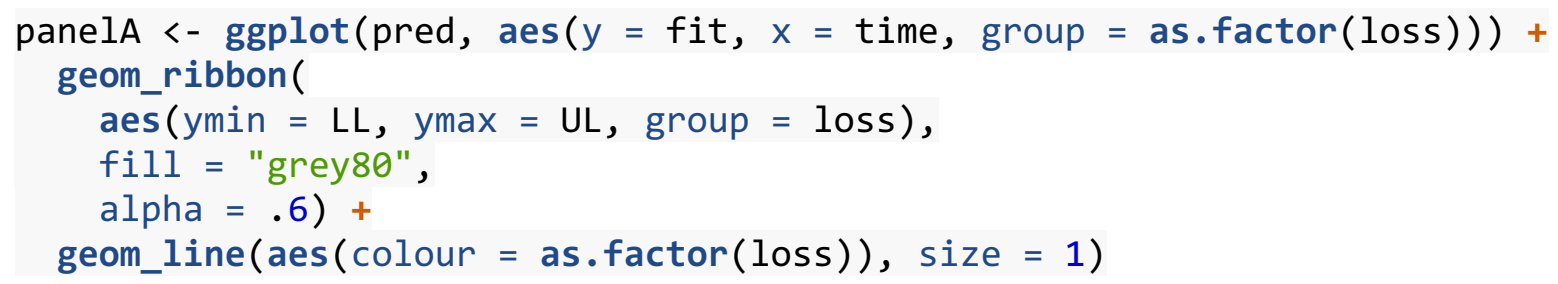

The above commands create the plot object. To render the plot, run a line calling up the completed plot object:

panelA

Your rendered plot should look something like the image in Figure 3, Panel A. By default, ggplot2 has selected a background theme, superimposed a reference grid, and displayed only the range of values along the axes for which we supplied data. The tick marks selected by default on the time axis also don't annotate the individual days of the study. We can resolve axis scaling issues by adding some additional plot commands and assigning the result to a new plot object, panelB. Here we use two scale functions to give each axis a label, control the range of values displayed, and rename the axes with more appropriate descriptive labels. The possible range of response options for the outcome variable (Likert-rated scores from 1 to 5) exceeds the range of the fitted values, so we can use the limits option to define upper and lower boundaries of the $\mathrm{Y}$ axis. Using the breaks option for the Day axis, we request a sequence of values starting at 1 , ending at 10 , and incrementing by 1 to display each study day on the axis:

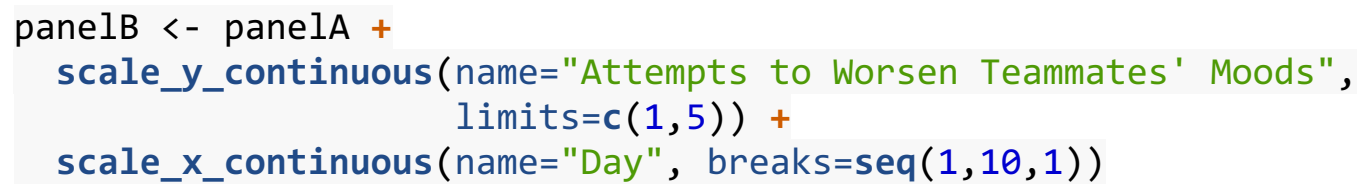




\section{Figure 3}

Interim Figures Created by Adding and Editing Plot Elements Step-by-Step
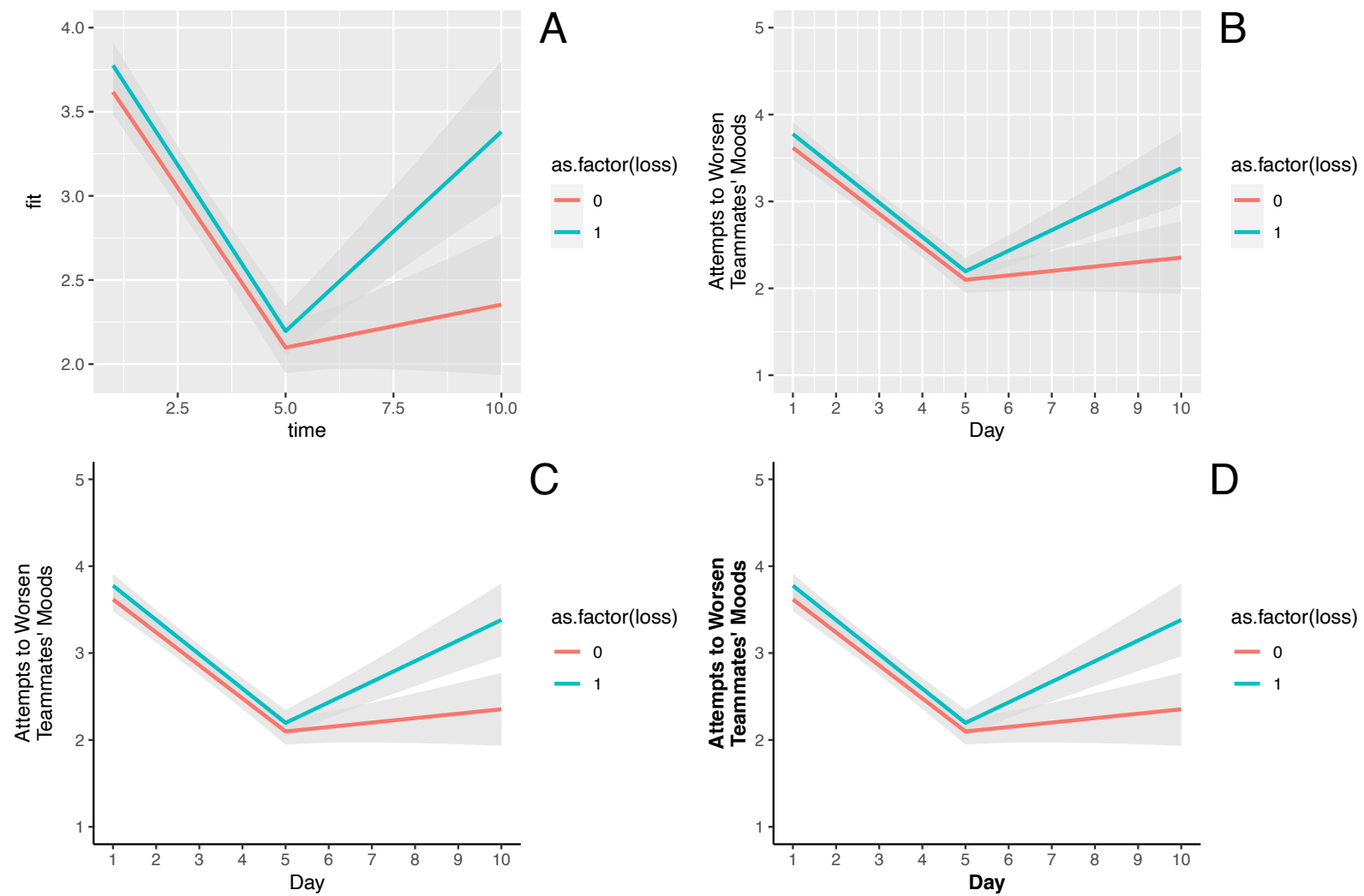

C
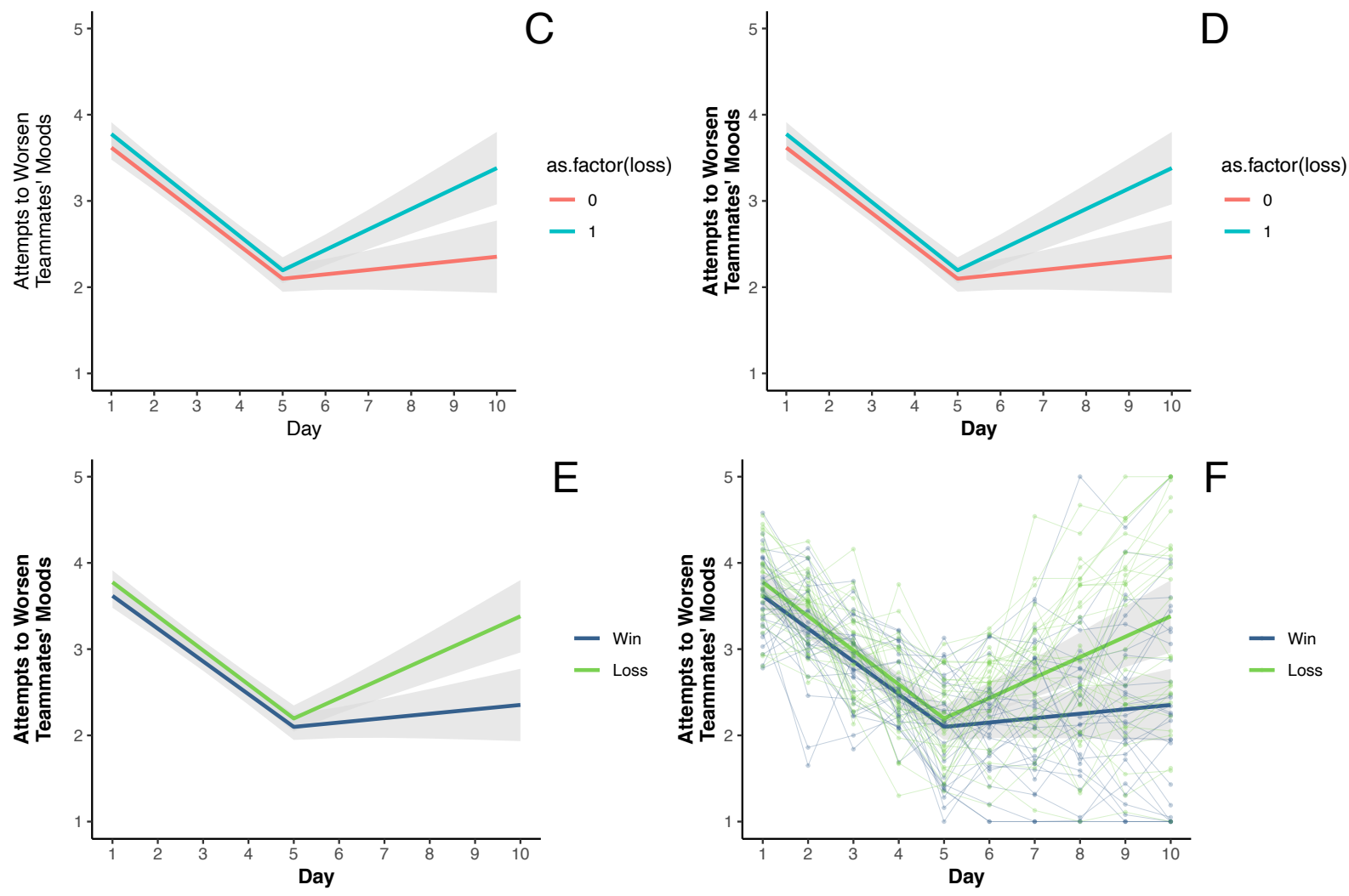

Note. To fit the plots into this multi-panel display, the $\mathrm{Y}$-axis label runs over two lines. This is accomplished by editing the name option to indicate a new line break $(\mathrm{n})$ in the middle of the label: name = "Attempts to Worsen\n Teammates' Moods" 
The rendered plot object appears in Figure 3, Panel B. This plot satisfies the goals of accurately displaying the trend lines with uncertainty, but the confidence bands are difficult to see because of the grey background. Plot themes are used to control the noninformative elements of the display, and ggplot2 includes several useful default themes (see https://ggplot2.tidyverse.org/reference/ggtheme.html for more details). In the interest of limiting chartjunk, I chose a fairly simple base theme that can be added to the existing plot and assigned to a new plot object, panelc:

panelC <- panelB +

theme_classic( )

Figure 3, Panel C shows that this plot is easier to read but could still be improved. The most prominent elements limiting the success of this plot are the font sizes of the axis labels, the lack of informative labels identifying the different trajectories, and the default color selection which is neither perceptually uniform nor suitable for readers with colorblindness. A small tweak with an extra theme command increases the font size and applies bold face to the axis labels, as shown in Figure 3, Panel D:

panelD <- panelC + theme(axis.title = element_text $($ face $=$ "bold", size=16) $)$

The line colors and legend labeling can be controlled within a single scale_colour command. The ggplot2 package includes numerous color palettes that can be referenced but an increasingly popular option is to use one of the palettes available in the viridis package for their aesthetic appeal, readability when printed in grayscale, and accessibility to readers with colorblindness. A scale_colour function for discrete variables from the viridis package adds to the plot a viridis color scale with legend labels, as shown in Figure 3, Panel E. The "begin" and "end" options can be used to select points on the viridis color gradient from which to draw color selections: 


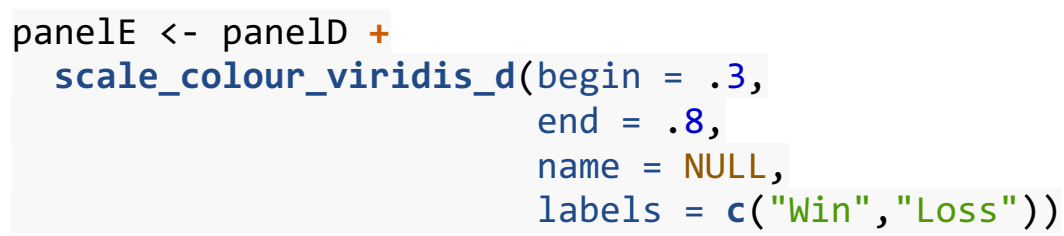

Try modifying these values between 0 and 1 to see how the rendered plot changes.

Depending on your needs and preferences, you may instead prefer to generate a grayscale or black-and-white plot. In that case, return to the code used to generate Figure 3, Panel A, replace the geom_line command with the following, and use scale_linetype (instead of scale_color) to control the legend labeling:

\#\# Alternative commands if black-and-white lines are preferred:

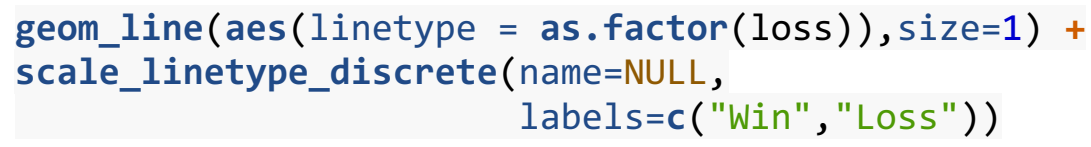

\section{Adding raw data to complement mean trajectories}

An important strategy for enhancing the transparency of the findings and the conditions in the data that led to the fitted trajectories is to plot the raw data alongside the group mean trajectories and confidence bands. Particularly where there are interactions, raw data can help to reveal links between variables, outlier influence, and nonlinearity that may not be apparent from the fitted lines alone (Tay et al., 2016). Raw data in a plot of repeated measures or longitudinal data calls for more than just a scatterplot. Most participants will have contributed multiple observations, and their observations are linked. Such data are well-represented graphically as a spaghetti plot, so named for giving the appearance on the Figure of a handful of spaghetti noodles tossed onto a flat surface. At the outset of this tutorial, we loaded a data file containing individual participant responses and assigned it to an object named athlete. We can directly reference that data in two new geom commands: geom_point() to overlay a scatterplot of participants' observed data and geom_line() to connect the observed data points. 
In prior geom commands, the identity of the data object was inherited from the initial ggplot ( ) command and written into the object panelA. We now need to identify a new data object from which to draw information. We also need to identify any aesthetic mappings that deviate from those listed in the initial ggplot( ) command. Here, the y axis variable is called "score". The critical option we use as an aesthetic mapping for geom_line is to identify a new grouping variable. Specifically, we want to plot a separate line for each value of "id" in the data. This option is what gives the "spaghetti" appearance to the raw data shown in Figure 3, Panel F. Other options are as before, and here I've chosen to make the lines and data points fairly small and transparent:

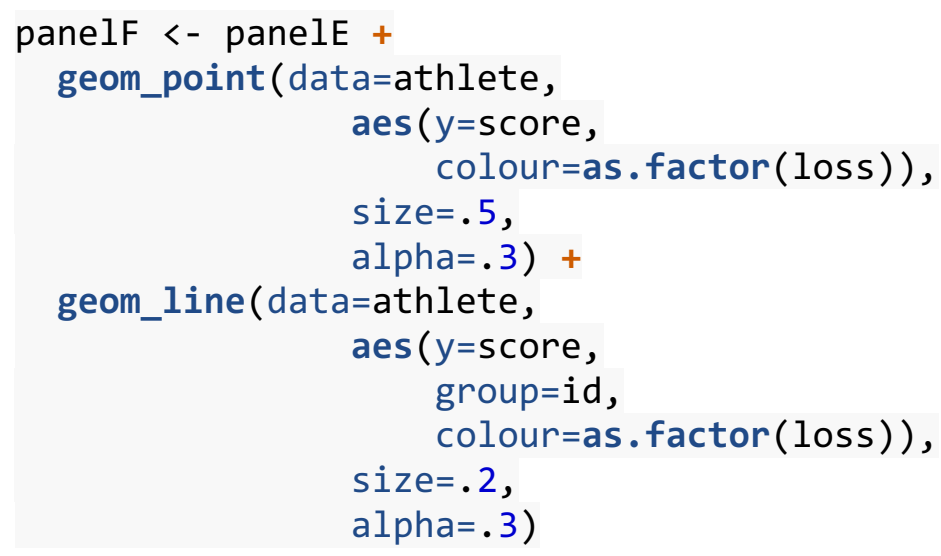

The layered structure to plots made with ggplot2 is immediately apparent. Since we added the raw data last, it appears on top of the mean trajectories and confidence bands. Raw data is more effective in the plot background where it doesn't occlude other plot elements. With that in mind, the commands below rebuild the entire figure pictured in Panel F of Figure 3, except that the geom commands to add the raw data are layered in first instead of last:

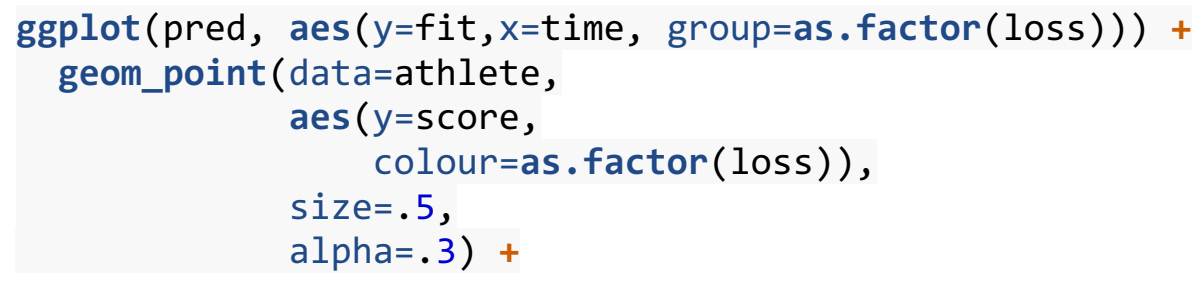




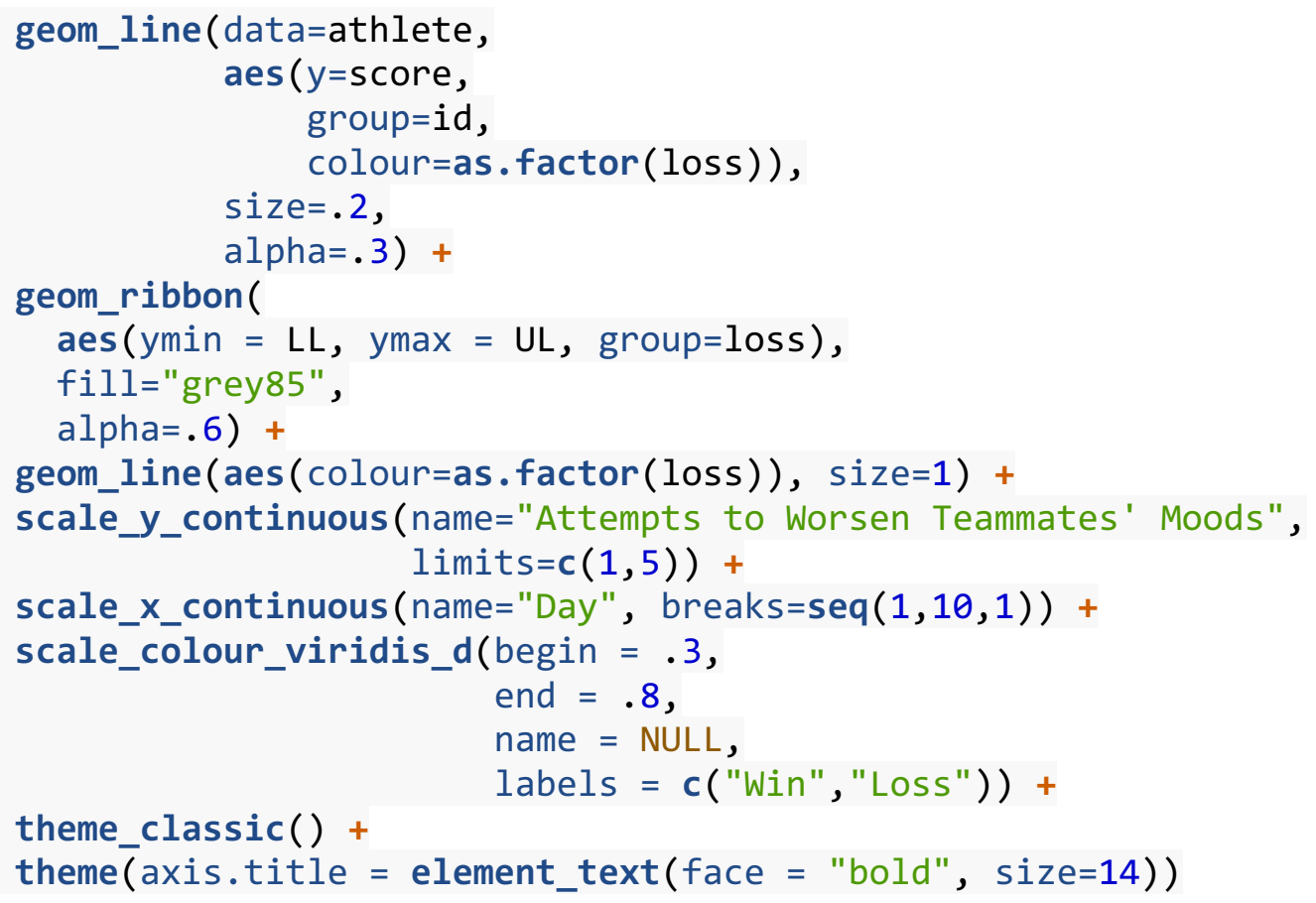

Figure 4 shows the final plot, which includes annotation to mark the location of the athletes' competition day after their Day 5 assessment but before their Day 6 assessment. Unlike Figure 1, it is clearer from this final plot that athletes from losing teams don't begin to meaningfully differentiate from their peers on winning teams until Day 7, when the trajectory confidence bands no longer overlap. The variability in individual trends evident from the raw data also highlights the degree of departure from the group mean trajectories - for example, some athletes from winning teams report attempts to worsen teammates' moods that exceed the means of athletes from losing teams.

Annotation can be used in addition or as an alternative to a figure legend, to highlight important plot elements or outliers, or to add brief descriptive content that might otherwise appear in a caption. Strategies for plot annotation are covered nicely in Chapter 5 of Healy (2019). 


\section{Figure 4}

Final Annotated Plot of Mean Trajectories for Athletes whose Teams Won and Lost their

Competitions, with Confidence Bands and Raw Data from Individual Athletes

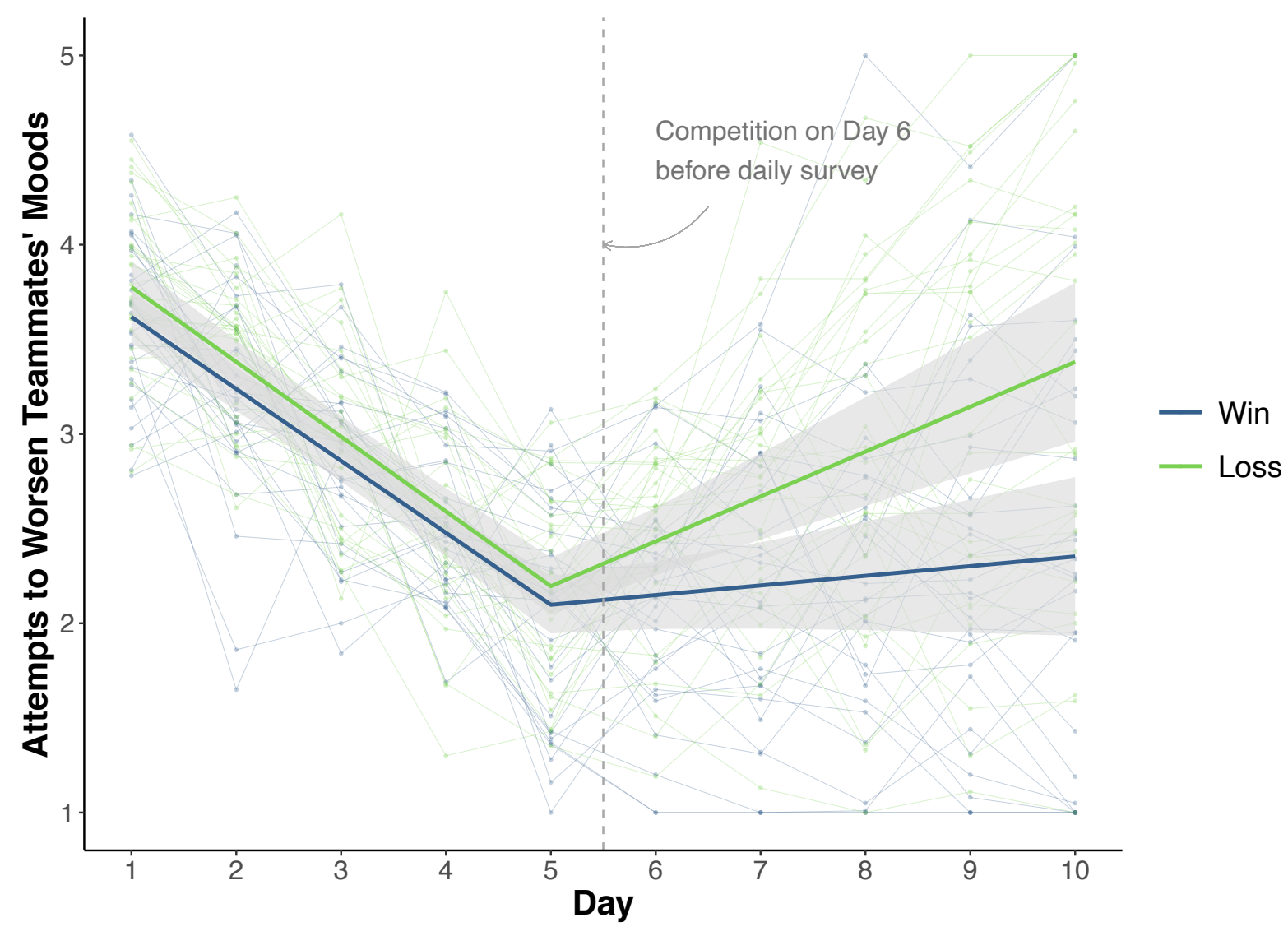

The following commands can be added to the code for Figure 4 (be sure to terminate any existing plot layers with a "+" symbol before adding new commands). First, a vertical line can be added anywhere on a plot canvas using the geom_vline function (see geom_hline if a horizontal line is desired) and indicating its location along the x-axis. The annotate function applies the "Competition Day" label to the plot, centered on the $\mathrm{x}$ and $\mathrm{y}$ coordinates given, with customizable size and color options. The geom_curve function draws a curved arrow, beginning and ending at the $\mathrm{x}$ and $\mathrm{y}$ coordinates given in the aesthetic mapping. Options for arrow length, 
thickness (size), color, and degree of curvature are all customizable and the selections shown below should be considered a starting point for experimenting with your own plots:

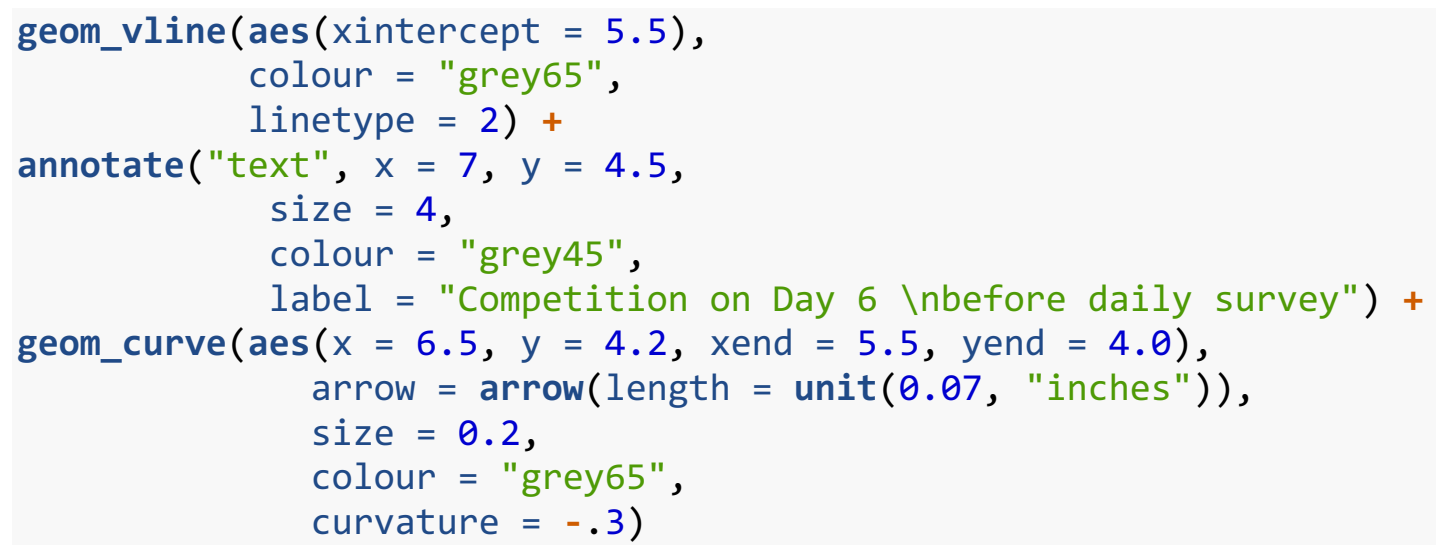

\section{Summary}

This tutorial provided a step-by-step guide to visualizing trajectories of mean-level change for studies modeling repeated measures data. This included a general-purpose technique for calculating delta-method composite standard errors of fitted values arising from a model, and commands for building a plot using $\mathrm{R}$ software that features confidence bands and raw data. A cursory glance through recent issues of Psychological Science suggests that plots featuring all of the above elements are rare (see Hittner et al., 2020 for an example). Plots of mean trajectories with no information about model uncertainty are still common (e.g., Jebb et al., 2020; Padovano et al., 2020), and those that include confidence intervals or bands often leave out raw data (e.g., Aichele et al., 2018). This tutorial resolves an important barrier to generating informative and transparent plots by decoupling data visualization from model development. Researchers performing analyses in any software can bring data and key output to the R programming environment to build accurate, beautiful, and scientifically transparent graphics depicting trajectories of change. 
Author Contributions

A. L. Howard is the sole author of this manuscript and is responsible for its content. She developed the idea, wrote the article, generated simulated data, wrote accompanying R scripts, and created all Figures shown here.

\section{Acknowledgements}

I am grateful to Drs. Erin Barker and Megan Lamb who assisted in testing the data and scripts in this manuscript and for providing helpful comments on an earlier draft. 


\section{References}

Aichele, S., Ghisletta, P., Corley, J., Pattie, A., Taylor, A. M., Starr, J. M., \& Deary, I. J. (2018). Fluid intelligence predicts change in depressive symptoms in later life: The Lothian birth cohort 1936. Psychological Science, 29(12), 1984-1995.

Aiken, L. \& West, S. G. (1991). Multiple regression: Testing and interpreting interactions. Sage Publications.

Bates, D., Maechler, M., Bolker, B., \& Walker, S. (2015). Fitting linear mixed-effects models using lme4. Journal of Statistical Software, 67(1), 1-48.

Bauer, D. J. \& Curran, P. J. (2005). Probing interactions in fixed and multilevel regression: Inferential and graphical techniques. Multivariate Behavioral Research, 40(3), 373-400.

Bryk, A. S., \& Raudenbush, S. W. (1987). Application of hierarchical linear models to assessing change. Psychological Bulletin, 101(1), 147-158.

Cumming, G. (2014). The New Statistics. Psychological Science, 25(1), 7-29.

Dorfman, A., Oakes, H., Santos, H. C. \& Grossmann, I. (2021). Self-distancing promotes positive emotional change after adversity: Evidence from a micro-longitudinal field experiment. Journal of Personality, 89, 132-144.

Flora, D. B. (2008). Specifying piecewise latent trajectory models for longitudinal data. Structural Equation Modeling, 15, 513-533.

Garnier, S. (2018). viridis: Default Color Maps from 'matplotlib'. R package version 0.5.1. https://CRAN.R-project.org/package= $=$ viridis

Healy, K. (2019). Data visualization: A practical introduction. Princeton University Press.

Hittner, E. F., Stephens, J. E., Turiano, N. A., Gerstorf, D., Lachman, M. E., \& Haase, C. M. (2020). Positive affect is associated with less memory decline: Evidence from a 9-year longitudinal study. Psychological Science, 31(11), 1386-1395. 
Jebb, A. T., Morrison, M., Tay, L., \& Diener, E. (2020). Subjective well-being around the world: Trends and predictors across the life span. Psychological Science, 31(3), 293-305.

Kuznetsova, A., Brockhoff, P. B., \& Christensen, R. H. B. (2017). 1merTest package: Tests in linear mixed effects models. Journal of Statistical Software, 82(13), 1-26.

Li, P. \& Redden, D. T. (2015). Comparing denominator degrees of freedom approximations for the generalized linear mixed model in analyzing binary outcome in small sample clusterrandomized trials. BMC Medical Research Methodology, 15:38, 1-12.

Long, J. A. (2019). interactions: Comprehensive, User-Friendly Toolkit for Probing Interactions. R package version 1.1.0, https://cran.r-project.org/package=interactions.

Luke, S. G. (2017). Evaluating significance in linear mixed-effects models in R. Behavior Research Methods, 49(4), 1494-1502.

Marsh, L. C., \& Cormier, D. R. (2002). Spline Regression Models. Sage Publications.

Meredith, W. \& Tisak, J. (1990). Latent curve analysis. Psychometrika, 55(1), 107-122.

Ogasawara, H. (1999). Standard errors for matrix correlations. Multivariate Behavioral Research, 34(1), 103-122.

Padovano, H. T., Janssen, T., Sokolovsky, A., \& Jackson, K. M. (2020). The altered course of learning: How alcohol outcome expectancies are shaped by first drinking experiences. Psychological Science, 31(12), 1573-1584.

Preacher, K. J., Curran, P. J., \& Bauer, D. J. (2006). Computational tools for probing interactions in multiple linear regression, multilevel modeling, and latent curve analysis. Journal of Educational and Behavioral Statistics, 31(4), 437-448.

Raykov, T. (2002). Analytic estimation of standard error and confidence interval for scale reliability. Multivariate Behavioral Research, 37(1), 89-103. 
R Core Team (2019). R: A language and environment for statistical computing. R Foundation for Statistical Computing, Vienna, Austria. URL https:/www.R-project.org/.

Rogosa, D. (1980). Comparing nonparallel regression lines. Psychological Bulletin, 88(2), 307321.

Satterthwaite, F. E. (1941). Synthesis of variance. Psychometrika, 6(5), 309-316.

Tamminen, K. A., Page-Gould, E., Schellenberg, B., Palmateer, T., Thai, S., Sabiston, C. M., \& Crocker, P. R. E. (2019). A daily diary study of interpersonal emotion regulation, the social environment, and team performance among university athletes. Psychology of Sport \& Exercise, 45, 101566.

Tay, L., Parrigon, S., Huang, Q. \& LeBreton, J. M. (2016). Graphical Descriptives: A way to improve data transparency and methodological rigor in psychology. Perspectives on Psychological Science, 11(5), 692-701.

Tufte, E. R. (1983). The visual display of quantitative information. Graphics Press.

Wickham, H. (2016). ggplot2: Elegant Graphics for Data Analysis. Springer-Verlag.

Wickham, H., François, R., Henry, L., \& Müller, K. (2021). dplyr: A grammar of data manipulation. R package version 1.0.5. https://CRAN.R-project.org/package=dplyr

Young, E. S., Farrell, A. K., Carlson, E. A., Englund, M. M., Miller, G. E., Gunnar, M. R., Roisman, G. I., \& Simpson, J. A. (2019). The dual impact of early and concurrent life stress on adults' diurnal cortisol patterns: A prospective study. Psychological Science, 30(5), 739747. 